\title{
Ensembles of Practice: Older Adults, Technology, and Loneliness \& Social Isolation in Rural Settings
}

\begin{abstract}
This paper considers whether, and if so how, ICT might play a role in addressing loneliness and social isolation for older adults in rural areas. We base our paper on a longitudinal co-creation study that has been undertaken in a rural location in the North of England. We adopt a practice perspective to examine the everyday practices of older adults as they sought out opportunities to address their desire for social connectedness and explore how technology might support them in those practices. Specifically, we argue that we need to identify the linking practices that enable participation in social connectedness through ICT. Based on this, we develop a model to guide future practice based studies and interventions.
\end{abstract}

\section{Introduction}

Europe's population of older adults, especially those living in rural areas, is growing steadily. As people are living longer, issues linked to reduced mobility, fewer opportunities for social interactions, worsening health and low income can, among others, lead to significant degrees of loneliness and social isolation. Broadly defined, loneliness refers to the perception of there being no significant other that is involved in their lives while social isolation refers to the limited quantity of social networks available to a person. While loneliness is experienced by all ages, it is especially pronounced in older adults. Approximately 3.8 million older adults over 65 in the UK live alone of which 70 per cent are women [1]. $51 \%$ of all people aged 75 and over live alone and approximately $6 \%$ of older adults leave their house once a week or less [1]. Loneliness is the cause of declining physical and mental health conditions such as obesity and depression. Consequently, addressing loneliness and social isolation is seen to lead to significant socio-economic benefits such as reducing the number of healthcare visits and allowing older adults to live independently in their own homes for longer. Loneliness is especially pronounced amongst older adults that reside in rural districts. A recent report estimated that $23 \%$ of the rural population are over retirement age compared to $18 \%$ in urban areas [1]. Further, the percentage of older adults living in rural areas is increasing at a greater rate than in urban areas.
Especially for the over $85 \mathrm{~s}$ who are expected to increase by $186 \%$ by 2028 in rural areas [2].

This paper will consider whether, and if so how, information and communication technologies (ICT) might play a role in addressing loneliness and social isolation for older adults in rural areas. We base our paper on a longitudinal co-creation study that has been undertaken in a rural location in the North of England (South Lakeland). It has a higher proportion of older adults than is average in the UK. Through a participatory action research approach, that included co-creation and action learning through reflective practice we have sought to understand what meaningful social connectedness is perceived to be and how older adults seek to access social connectedness. The specific aim being to explore a possible technological intervention that could assist in addressing this important issue. We did this by investigating the practices of older adults - what they do, where they go, and who they do it with, through a number of participatory action research methods. Through this co-research we discovered that we needed to consider the ensemble of situated and entangled practices that are constitutive of the possibilities for older adults to participate in social connectedness.

This paper is structured as follows. First, we review the literature on older adults, ICT and rural settings. Next, we outline our methodology and methods. Following this, we draw on our primary research findings to argue that addressing loneliness and social isolation requires us to develop technology around key linking practices. Finally, we offer some conclusions and implications.

\section{Literature review: meaningful access?}

Given the prevalence and consequences of loneliness and social isolation in rural districts, one would imagine that some form of technological oriented interventions might already have been considered and experimented with. What does the literature say? Poscia et al [3] published a review on interventions to tackle loneliness and social isolation in older adults. Of the 15 studies they analysed, only four examined ICT solutions and only one of these examined internet usage [4]. Other approaches such as Austin et al [5] made use of technology to monitor loneliness levels. However, they did not consider the 
possible use of technology in alleviating it. Hill et al [6] argued that technology has the potential to reduce loneliness and social isolation, however they claim it should not be used in replacing direct social contact but should complement 'face-to-face' interactions. A view we would agree with.

With regards to older adults use of ICT, Niehaves and Plattfaut [7] found that a large number of older adults are still reluctant to engage with ICT. They found that although age is a factor (older elderly people were less likely to use the internet than younger elderly), there are also other social-demographic factors to consider. Rickman et al [8] found that the job that older adults did prior to retirement was central in understanding the degree of participation in digital health interventions.

Fox and Connolly [9] studied the barriers to older adult use of mobile technology and found that many older adults had concerns about privacy. Barnard et al [10] highlighted the importance of support to older adults when they are learning to use new mobile devices (i.e. tablets). They identified four factors in the system (transparency, affordance, feedback, and error recovery) that influence how easy it is for older adults to learn how to use it.

The term 'digital divide' is used to identify those groups that are less likely to have access to the internet. Schreurs et al [11] argue that the 'grey divide' is characterized by the lack of digital literacy skills. They claim this is the main factor preventing older adults to make greater use of digital devices and services. Rockmann and Gewald [8] identified a number of different traits amongst older adults that they claim can explain the extent and nature of their use of the internet. Sipior et al [12] highlight that it is important to work with communities where acceptance of technology is low to increase their levels of adoption before any digital service solution can be successful.

In relation to rural settings, the focus of this paper. Philip et al [13] studied a urban-rural digital divide, where access to the internet, either through broadband or mobile networks is limited due to the lack of infrastructure in remote rural areas. Ashmore et al [14] explored community-led approaches to expand the reach of broadband in remote rural areas. Boase [15] identified demographic factors such as an older population and a lower level of education in rural areas when compared to urban ones, influenced the level of internet use. Saunders'[16] research identified the cost of the equipment, physical difficulties in using computers and "fear of looking stupid" as other barriers. Hakkarainen's [17] study of rural communities in Finland found that many older adults did not trust technology nor did they see it having any use for them. Similarly, Hodge at al [18] found that older adults did not find many services online that they could use.

However not all discussion around older adults and ICT use focus on exclusion. Suopajarvi's [19] study of on older adults ICT users found they lacked the skills to use technology extensively. Despite this, they still found that it had become part of their every day practices. Similarly, Quan-Haase et al.'s [20] research on 'digital seniors' found they were adopting new routines through their use of ICT. They found that some were 'hybrid digital practices' where new practices were merging with established practices. Bozan et al [21] found that older adults adoption and use of digital health technologies was in part influenced by their healthcare providers' advice, but was also greatly influenced by people in their family / social networks that they respected and trusted. Further, mobile devices in the delivery of digital services has the potential to provide personalized services [12]. As mobile devices are used by one person and enable location-based applications, they can be used to identify individual users in service delivery.

What the literature review has highlighted is that technology may have a role to play in addressing loneliness and social isolation among older adults in rural districts. It has highlighted that access to the internet, technology design, as well as the limited competencies in older adults needs to be addressed. While not disputing these findings, we would suggest that they need to be seen in a broader, or perhaps different perspective. Specifically, we would suggest that appropriate and meaningful technology use (or mediation) emerges in and through situated and entangled practices in the flow of everyday life. As such a practice oriented lens is required. One that:

"more easily accommodates people's situated use of dynamic technologies because it makes no assumptions about the stability, predictability, or relative completeness of the technologies. Instead, the focus is on what structures [or practices] emerge as people interact recurrently with whatever properties of the technology are at hand..." (p. 407) [22]

This situated practice approach suggests first that studies and interventions in loneliness and social isolation through the use of ICT needs to remain situated in the daily lives of the older adults for it to be meaningful and relevant. Thus, what is important is to understand what they do, where they go and who they do it with.

Second, the practice lens focuses our attention on the ensemble of interconnected sociomaterial practices [23]. Rather than focusing on the individual or on the technology, the phenomenon of concern is the ensemble of situated and entangled sociomaterial 
practices (involving both human and technological practices) that come into play as older adults attempt to deal with loneliness and social isolation in their daily lives. These practices evolve over time. The situated ensemble of sociomaterial practices are accomplished in and through the interaction of many different elements, for example the material infrastructure, knowledge and competencies, and common understandings and meanings [24]. A practice approach provides a way to explore how practices have evolved and changed over time and how people become involved, or not, in these practices [25]. What is specifically interesting, for us, is how, why or why not an ensemble of practices come about, and take hold, and for who.

\section{Empirical context and research methodology}

The empirical context. The South Lakeland District is part of the county of Cumbria and covers 600 square miles in the North West of England. It is a predominantly rural area that includes much of the Lake District and the north-western parts of the Yorkshire Dales. The district consists of four main urban areas many villages, hamlets and isolated properties. It has a population of 103,500 (in June 2012) and a population density of 68 inhabitants per $\mathrm{km} 2$ (Office for National Statistics, 2011). Ninety-nine percent $(99 \%)$ of citizens resident in the South Lakes were born in the UK and $33 \%$ of the population are aged over 60 , compared with a national average of $23 \%$. There is a projected $47.5 \%$ increase in residents aged over 65 from 2012 to 2037 (12.500 persons). In 2011, 20\% of the South Lakeland population was retired, compared to 14\% for England (Census 2011). Many older citizens in South Lakeland live alone. Census figures show that there are 7,600 one-person households aged 65 and over and 5,802 family households where all members are aged 65 and over in the district (Office for National Statistics, 2011).

The project. The project we are considering here is one part of an EU funded project to develop (through co-creation approaches) digital solutions that can use open data to support the delivery of public services to older adults. The project could be roughly divided in two phases: an exploratory phase and a development phase. The initial phase had emphasis on engaging participants and stakeholders, interacting with them to understanding the context of the problem to be addressed and co-creating together a new understanding of the problem and a vision/paper prototype for a possible solution. The second phase was centred on developing the prototype of the apps at iterative steps, tackling different issues of interface, use and content. As new aspect of the apps was improved, new ideas to develop other areas emerged from our cocreation workshops.

The co-creation of the app and service, as well as defining what open data would be used happened concomitantly. Once the working prototype of the app was working reliably, the interest of the co-creators turned to how people would use it in their daily lives. Having a working prototype of the app made the discussions around use more concrete and we were able to move forward with the plans for service implementation and delivery. The focus of the cocreation was not just on developing technology, but also on co-creating ideas for service implementation where the co-created apps could be used as tools to fulfil the overall goal of the project which was help tackle social isolation and loneliness.

Our research orientation. Our research practice is rooted the participatory action research tradition [31]. Practically this was implemented through two interrelated methodologies: co-creation (the doing of the project) and reflective practice (learning from our doing). Co-creation is a participatory process and practice rooted in design research approaches, such as co-design [30], research through design [29] and action-research [35, 33]. Reflective practice is the process of learning through direct experience where practitioners observe their own practices, reflect on them, and discern what can be learnt from them in order to become progressively more competent and informed about these practices [32, 34].

The enactment of our participatory action research methodology consisted of a combination of co-creation workshops together with reflective qualitative methods such as reflective observations, post-workshop debriefings, interviews, and focus groups. The fieldwork was carried out from March 2016 to July 2018 and included 35 interviews, 6 focus groups and 26 co-creation workshops during this period, as well as meetings with local service providers. We also adopted a longitudinal approach in our co-creation workshops, working with the same core group of 7 older adults for over 2 years. In addition, we conducted 2 user-testing sessions with older adults that did not take part in the co-creation workshops and a pilot study in partnership with Age UK South Lakeland to trial a scheme where older adults were loaned a tablet containing the Mobile Age Social Connectedness apps. 9 Age UK volunteers took part in this trial. Over 80 people, including older adults, intermediaries (those people such as family, friends, support workers and carers that assisted older adults in accessing mobile technologies and the internet), service providers and researchers participated in the research. In the context of our research, the term 
older adults refer to people that are aged 55 or older. Demographic data from participants was collected on certain activities such as the co-creation workshops, user testing and semi-structured interviews, although we were not able to collect this type of data in observations and some informal discussions. We collected demographic information on 34 older adults ranging from 55-89 years old with an average age of 69.3. The percentage of females to males were approximately 2: 1 (22 female and 12 male). Only 3 of the participants declared to have a disability.

Qualitative data from interviews, focus groups, reflective observations and debrief meetings were captured in notes and voice recordings. The analysis of this qualitative data was also participatory in that the researchers would identify certain themes and then test these with the older adult participants - encouraging them to add to, or amend, these according to their own experience. The analysis identified themes that were used in understanding the context of loneliness and social isolation as well as the everyday practices of the local older adult population in dealing with mobile technology and engaging in social participation. The insights gained from the participation in the workshops--and the ongoing analysis of the qualitative data-informed the ongoing development of the cocreation workshops as the co-creation process unfolded. In other words, the action research was both formative and summative.

Co-creating workshops. Each co-creation workshop lasted around two hours. University researchers, including facilitators and developers, co-created together with older adults, and in a couple of instances, with representatives from local service providers, such as local government and NGOs and intermediaries. Methods employed in the workshops included design research tools such as probes, personas, prototyping and activity sheets and cards, as well as group discussions. Through the regular co-creation workshops, ideas were developed from conception to the creation of demonstrator mobile applications, passing through a series of prototypes and iterations on the design of the applications and service scenarios. Data from workshops were captured through the activities and the discussion were often summarized in notes and discussed with participants. These findings were also used in the various iterations of the app development and were considered in the postworkshop debriefings. Thus, co-creation workshops acted both as co-development and co-research opportunities.

\section{Ensembles of sociomaterial practices}

When we reviewed the literature above we suggested that this literature tended to focus on specific aspects (of users, or technology, or use) without considering the ensemble of sociomaterial practices that enact the condition of possibility for such use to be possible and make sense. In this section, we will explore if mobile devices such as phones and tablets have a role to play in addressing loneliness and social isolation amongst older adults in rural districts. Specifically, we will seek to ascertain what sociomaterial practices do older adults endeavour to address their social connectedness, and second, what is the ensemble of sociomaterial practices of which they are part of? For the sake of brevity, we will refer to sociomaterial practices simply as 'ensembles of practice' but this always implies an entangled ensemble of both human and technological practices.

This practice perspective underpins Figure (1). As Pantzer and Shove [26] highlight a practice perspective requires us to "concentrate on networks of practice and on relations between practices distributed across time and space" (p.20). Such a view argues that we need to focus on the interdependence of different practices. By doing this it allows us to identify when these interlinking practices are enabled or broken. What it suggests is that we need to attend to the enabling linking practices pertaining to what older adults in rural districts do as they seek out opportunities for social connectedness. In broad terms, what enabling linking practices refers to is the practical ways these practices become interwoven as part of practical activity. Conversely, how when specific practices are missing, participation is curtailed or limited.

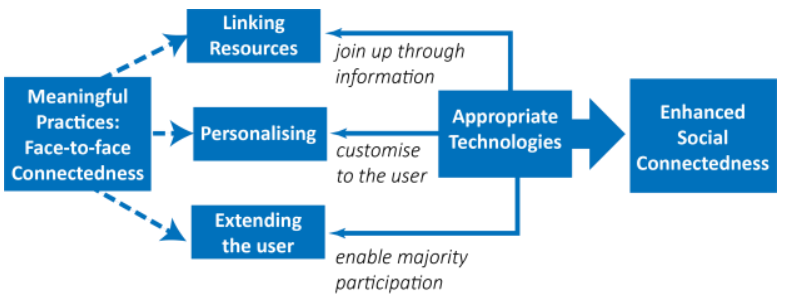

Figure 1. Enabling linking practices: enabling social connectedness through ICT

What this practice perspective leads us to argue is that developing appropriate technologies to address loneliness and social isolation needs first to attend to the meaningful practices older adults participate in as they seek out and engage in social connectedness. These, we will suggest, are those that they perceive to be especially meaningful. Second, we argue that what technology can do is to attend to the linking resources or practices, namely those dimensions that cumulatively need to be put in place for social 
participation to become possible. Third, we will argue that we need to personalize the information about the social connectedness opportunities and the linking resources to reduce the cognitive burden on older adults. Finally, we argue that technologies need to be designed not only for the competent older adult user but also for those with varying abilities, degrees of connectivity and none. We outline each of these arguments in turn in this section.

\subsection{Meaningful Practices: Enhancing face-to- face group social connectedness}

Practice theorists, Pantzar and Shove [26] highlight that it is crucial to identify those practices that are routine / sought out in relation to social connectedness. By doing this, we can identify what are especially meaningful forms of social connectedness. We found that older adults routinely sought out opportunities for face-to-face social connectedness primarily through attending social events run by public and third sector organisations or through volunteering [6]. Take the indicative example of Jack who is 80 years old and lives in a rural village. His wife has had a spinal stroke recently and does not like him leaving her alone at home for more than a few hours each day. He actively seeks out opportunities to get out of his house each day through joining exercise classes and by organising a men's monthly lunch. This gives him a chance to relax and alleviate the burden of care. What such an example illustrates, as does the literature, is that combating loneliness and social isolation is most effective by enabling face-to-face social connectedness [6].

We found that initiatives to address loneliness and social isolation are most effective when targeted at groups rather than individuals. Initiatives that prove especially beneficial include regular activities such as social events, education, and exercise classes. Volunteering, as with Jack, is also an important strategy for older adults in their attempts to live independently. Indeed, what was initially surprising to us was that all our core older adult co-creators volunteered at least once a week. They saw it as a way to stay socially connected and to feel valued. Of course, this was also a key reason for why they enrolled as co-creators. The importance of volunteering highlights that older adults themselves might play a vital role in setting up and operationalising projects to address loneliness and social isolation.

When the possibility of technology was discussed in relation to loneliness and social isolation, none of the older adults perceived a technology as a solution to social isolation as George, 78 years old widower who lives in rural village location exemplified:
"I am a basic technology user. Few of my friends have access to the internet. What we all want to do is to meet other people and get out of our own homes more. I cannot see how technology might help me or them do this. In fact, we fear that this may replace face-to-face opportunities for contact."

Even amongst those that had used video calls and social media, they were all adamant that this could not be a replacement for face-to-face social connectedness.

What does such an understanding of meaningful forms of social interaction suggest for the possibility of information technology to better facilitate social connectedness? Shove [27] used the example of digital photography to ask the question which practices remained static and which have changed? She highlights that elements of meaning have remained rather static - people still want to take pictures and the aesthetic conventions such as not chopping heads out of a picture have remained the same. However, the process of filing and modifying images was now done differently through digital means. This example resonates well with our findings. The meaningful practice of addressing loneliness and social isolation has remained static. Meaningful social connectedness thus was considered primarily face-to-face group based. Digital communication such as video conferencing or social media were not considered as being especially meaningful. However, as we will show, their long-established strategies that they drew on to find out about what is going on and how to do things are being disrupted by technology.

Technological alternatives were not possible for many older adults in the district due to their lack of internet access and / or competence in ICT. At the start of our research, only $16 \%$ of Age UK members surveyed had access to the internet.

We detected considerable negativity and distrust towards technology. All of them either had been or knew someone that had been scammed. Thus, they were cautious about using technology. They were all critical of the trend for more and more government services and information being moved online. They were also critical of computer-based call centres. They found these difficult or impossible to access. They liked to have face-to-face contact with someone they knew, as the person would often suggest other activities or services to the older adult. Alternatively, they would rely on finding out about events through their friendship network. In summary, they were either experiencing, or fearing, that the links that were crucial to their long-established strategies (and networks) for finding out what was going on were becoming broken. This meant that their social connected practices could no longer be reproduced, leading to social isolation. 


\subsection{Linking resources and practices}

To better understand this fear of their links being broken we will initially consider how information about social events was provided to them by public, private and third sector organisations. Many NGOs would provide information about social events and volunteering opportunities primarily in hardcopy (NGO offices, village halls, doctors or shop notice boards etc). Printed bus and train timetables would be made available in hardcopy at bus and train stations. Any community bus timetables would typically be posted in shop windows, parish halls etc.

Hard copy information was the most typical way of finding out about social opportunities by older adults. For example, Julie relies on printed adverts in public places such as libraries, doctor's waiting rooms or post offices. She also relies on her social circle for word-ofmouth information on up-coming events. However, some of the organisations had moved to also distributing their information online. The District Council had an events portal which included its theatres and sports facilities. The private sector transport providers had web pages and apps available to access their timetables. This was especially useful when timetables changed (as they often did). Some older adults sought out information online. For example, Mary, who is very comfortable with using IT, seeks out information about social activities on the websites of local organisations and uses online timetables. Differing organisations and older adults were thus providing and seeking information in a mixed mode of physical and digital data.

What most older adults had in common was that they planned their week ahead to build in opportunities for social connectedness. They did this for several reasons. First, planning the week was itself enjoyable exercise. They said that by reviewing different opportunities reassured them that they had a full week ahead to look forward to. Indeed, several of our older adults explained knowing they had things to do next week was just as important as doing the activities. As Margaret, a 78 years old widow who lives in a rural village location explained:

"What I want to do is to keep active both physically and mentally. I actively seek out social events such as fitness classes, training on computers and knitting groups. I also like to find out opportunities to volunteer. It is difficult to find out what is taking place in all the different villages and in the main town. If I do find out something is going on, I then need to find out how to get there, what the facilities are, what the weather will be and who else might be going."
What the quote above also highlights is that being socially connected required more than just knowing what is taking place, it also requires us to know how to get there, the facilities on route and at the location of the event. What she is pointing to are the important and often interconnected conditions that provided for face-to-face access. In our case, planning the week in this rural location comprised of four linking resources, transportation, weather, and daylight and toilet facilities. Transportation in rural settings is a significant logistical issue. Public transportation is especially important for older adults as many no longer have access to their own cars. Unlike in cities where there are trains and buses leaving every 5 or 10 minutes and hubs allowing for multiple connections, in rural settings there are often very limited services each day and few possibilities for connecting buses. Thus, many of the locations where social events take place were inaccessible in practice. Information about timetables is provided online by the national bus and rail organisations. However, as noted previously, older adults still rely on printed timetables. Additionally, transport services are increasingly being operated by new providers such as social enterprises as a response to the cuts in public transportation. These services are often only run at set times two or three days a week. Information is typically provided through posters and a website. Even for those older adults that had a car and would drive, there were still other constraints. Nina lives in a small remote location and she explained that some of the routes she had to take were difficult to drive during winter months.

Other important dimensions to becoming socially connected in this rural setting included knowing the weather forecast for a day and time in advance. Additionally, many older adults like to be home before darkness falls for safety reasons. Nor do they like traveling on country roads in the dark. This is especially so if they are driving themselves, but this also applies to users of public transport. Older adults did not feel safe walking on pavements or at the side of country roads after dark. Further, many older adults want to know the gradients and the route they will have to walk from the bus stop, or the car park, to the social event. They also like to know about toilet facilities on route. These elements (transport, weather, daylight and toilets) taken together, are all key linking practices and resources to facilitate face-to-face connectedness. Without these linking practices and resources, attending to face-to-face social connectedness would be difficult and potentially prohibitive. Thus, while the meaning of social connectedness remained static, it was the ways in which people accessed (and provided) information had started to change. To draw on Pantzer and Shove [26] we would suggest that technology 
could create new possibilities for social connectedness by linking elements into meaningful ensembles (or networks) of practice.

How might information technology build on such insights? Opening data would allow social connectedness opportunities to be publicized and shared. There are many NGOs operating in the district, some had opened-up their data, but they all operated with different data standards. Joining up information about the linking resources would alleviate the significant amount of practical cognitive and logistical activities (or practices) required to participate in social events. For example, identifying the correct bus (if there is one) and a connection (if needed) that will allow them to travel to the event on time, and a return journey, etc. Searching from site to site and mixing between paper and digital information is a key practical and cognitive challenge for older adults. Technology can aggregate, curate, and open-up information (from a variety of sources). Linking resources through relevant information, in a practical and situated manner is thus fundamental to addressing loneliness and social isolation.

\subsection{Personalising the service}

Our case and the literature highlights that the circumstances of each older adult are different and will change throughout old age. Public and third sector organisations (including those in South Lakeland) review the changing needs of older adults through their later life. First, the location of the older adult and the proximity of services, shops, family and friends. Second, the availability of transportation (private and third sector) and the associated costs. Third, life transitions such as bereavement, being a carer, and retirement are seen to be key risk factors that can result in an ever-decreasing quantity of social contacts and a declining psychological state. Fourth, deteriorating health conditions. And, fifth, their financial health, which might limit social connectedness. These dimensions act as a user profile that is updated as changes occur for each older adult. In our case, this was a paper-based record that would then be typed up and uploaded into a database. Towards the end of our fieldwork, there was work underway for the assessment to be shared between the different NGOs in the district. What this practice highlights was the importance of attending to the specificity of each older adult. How might ICT support this?

When we explored searching for information with our older adults, they explained they often found it difficult to search for information due to the volume, the number of different organisations providing the information, their lack of familiarity with the differencing search engines the sites employed and, with technology and the internet more broadly. We found that it was a significant cognitive burden on older adults to find out relevant and appropriately formatted information about what was going on and how they might get there etc. Tailoring the information provided to each older adult based on their individual profile would thus be beneficial [12].

We found in our workshops that what mattered was the perceptions of individual older adults about what they thought they were able to do. They claimed that their profiles were subjective and thus should be set by the older adults themselves. For example, rather than stating they suffered from a debilitating form of arthritis, instead they preferred to say that they could walk for 15 minutes, they wanted to see routes without steps etc. For older adults, the user profile should help them select the appropriate choices. They explained that two people with similar mobility issues could have very different perceptions of their limitations or of what they were willing to do. For example, while Annie would only feel confident, leaving the house with a carer to assist her, Pat preferred to be independent, use a scooter, and select venues herself with no steps. Similarly, while incomes vary greatly between older adults, they wanted to see all events regardless of cost. It allowed them to look ahead and save up for events that they were particularly interested in. It highlights the importance of older adults being able to access their profile themselves.

Developing an individual user profile that will match the services and information to the specific conditionality's of the older adults will greatly reduce the cognitive burden and enhance the possibilities for social connectedness. Further, it is also important for it to reflect the changes in circumstances that odder adults encounter. Pantzar and Shove [26] highlight, elements of practice are not static, they are constantly on the move. The conditionality's for each older adult can change temporarily (e.g. hospital discharge) or permanently through bereavement or giving up a driving licence for example. Thus, customising to the individual user needs to be ongoing. We found that NGOs in South Lakeland regularly reviewed the changing needs of their clients, which suggests that there is a role for them as well as government and health professionals to review and discuss their profiles considering each older adult's changing circumstance.

\subsection{Extending the user}

The final dimension that we will explore in relation to the development of technology to address loneliness and social isolation relates to the user. While there were some older adults that were advanced technology 
users, the current percentage of older adults in the district that make use of information technology and internet access was limited to only $17 \%$. Up only $1 \%$ in two years. Nor was there the prospect that this might change significantly in the short or medium term. There are a myriad of reasons for this low use of the internet in this region such as cost, poor/intermittent broadband coverage, etc. As with other rural areas, many of our older adults had never used computers and technology in their working lives and many do not see any benefits of learning and using it now $[15,17,8]$. Consequently, they do not know if there is any content on the internet that is relevant to them $[12,18]$. Nor could they make the association between the possibilities of technology and/to a greater social connectedness. Thus, returning to Pantzar and Shove's finding that many people could not participate in digital photography, as they did not have the relevant ensemble of practices (filing, manipulating and printing images) to make use of a digital camera, how might we think about developing a technological artefact that addresses the majority of older adults?

Amongst those that have used technology, many receive help and support from friends and family. Similarly, we interviewed family members who explained they would help their grandparents with technology. As Jack explains:

"My family and friends often use technology to find out things for me when I am stuck. However, most of my friends do not have internet access. They did not use technology in their working lives, as they were either farmers or manual workers. They still just ring people up or ask their children or grandchildren to use technology for them."

Our study thus found that their normal practice would be to seek help from intermediaries such as family and friends. However, seeking help also extends to carers, neighbors, and NGO \& government staff to routinely access the internet to find out relevant information [21]. Gabriel, a support worker told us he often searched for information on services and activities for his clients. Our study revealed that intermediaries often accessed relevant information for them based on a series of questions (such as 'what do you feel like doing tomorrow? Do you want to go to the coffee morning? And so forth). Given their responses, they would then access the relevant information (and resources) about these possibilities on their behalf. Thus, this practice — of finding out and linking — already exists in the interactions between older adults and intermediaries.

Crucially, these intermediaries are the people that have the necessary ensemble of practices, as well as access to the internet, that is required if technology is to be beneficial. How might this already available ensemble of practices be made accessible to the majority of older adults that could not be users of the technology themselves? ICT enabled practices (a combination of mobile technologies, open data and user profiles) can transform this practice for both the older adult and the intermediary. For example, the profile of the older adult can be shared (with their permission) with intermediaries that can use the apps to search for opportunities for social interaction on their behalf. Extending 'the older adult user' through the intermediary means that the production of digital and personalisable linking practices (and associated opportunities for connectedness) is available to all older adults regardless of their degrees of digital connectedness. One might say to connect in order to connect.

\section{Co-creating appropriate technologies}

In the section above, we outline some of the insights that we have gained through our participatory action research enacted through a co-creation process. What did this action research produce? Our action research project has co-created a fully functioning demonstrator being piloted in South Lakeland. The apps developed in the project include a launch portal, events, services and volunteering app. A user profile permits the older adult to select their preferences relating to transportation, how far and for how long they want to travel and sets a limit to cost of events. A user can request to view transportation options and routes to the event on an age friendly map (including the location of nearby toilets and benches). A calendar allows the user to organise their schedule. Importantly, the app can also be accessed by an intermediary who can search on behalf of the older adult and review their user profile. If required, the intermediary can then print out their calendar and activities for the week ahead. This takes away the necessity for the older adult themselves to be the user and / or have internet access themselves. It draws on open data about events provided by local government and third sector organisations.

Going forward, private sector organisations can also include their information as long as it is provided in an open format. It also includes volunteering opportunities that are provided by the NGOs in the district. In relation to transport, it draws on private sector rail and bus transport providers open data. It will be expanded to include the community transport services when they are able to provide this. For car users, we draw on open data that highlights public car parks. The app also draws on open data pertaining to subset and sunrise (darkness) and the weather. There is also local open data available about a scheme where 
traders provide free access to toilet facilities in their shops. Government open data about public toilet facilities and the location of benches is also provided. What this allows older adults to do thus is to have joined up information provided to them so as to help plan their week and details of how to attend social events or volunteering opportunities.

To recognize that much information remains printed in hard copy (e.g. posters on notice boards in doctors or post offices) around the district we have also developed an app called contribute a posted which allows users to upload images (photographs or scanned) of posters about local events that might not be listed in digital format (i.e. local car boot sale). This is being piloted, as it will require a moderator to review and maintain it. The key point is: the technological solutions that were co-created follow existing practices, maintaining their meaning to older adults but providing new ways to perform then.

\section{Conclusions and Implications}

What we have argued is that developing technologies to address loneliness and social isolation needs to be done by adopting a practice lens. This relational and systemic approach views practical activity as an ensemble of situated and interconnected practices. What is specifically interesting, is how, why or why not a particular ensemble of practices come about, and take hold, and for who. A situated, practice-based approach demands that we, as researchers, pay closer attention to the 'everyday' [25], and that we focus our attention on the everyday situated practices. As Pantzar and Shove[28] remind us we need to question what objects are being designed for, the practices of which they are part of, and if these practices can somehow be steered / changed?

Our practice-based analysis provides implications for technological developments that address loneliness and social isolation. First, it suggests designers need to attend to those meaningful practices that people seek out and value-which is face-to-face group social interaction. Second, we would suggest that they need to identify the necessary, interlocking, and enabling linking practices and resources required by older adults to be able to participate in meaningful face-to-face social connectedness. Such a view starts with existing practices and allows for the study and subsequent intervention into the ways in which technology might enable (or disable) certain ensembles of practices. Third, we need a strategy to encourage and support private, public and third sector organisations to open-up data. Open data needs to be in a format that allows for it to be shared between these organisations. Expertise, time, financing and infrastructure will be key resources that will be needed to bring this about. This is likely to be especially so for small NGOs (such as parish groups) and private sector organisations (such as fitness classes), to be able to participate. Fourth, designers need to identify how information can support the key linking resources. Fifth, personalizing the information based on a user profile can assist in reducing the cognitive burden for older adults. Finally, we need to design for the majority of older adults. This requires extending the constellation of practice to include intermediaries such as relatives, friends etc. (and their infrastructure). Thus, traditional approaches that focus on training individual users or on interface design only comprise of a small component of this constellation of practice. Indeed, our proposed intervention is itself only a part of a much wider ensemble of interventions such as befriending, assisted living and other social care and healthcare interventions - a wider ensemble of practice. Understanding and including this already meaningful ensemble of practices is central to any technological intervention aimed at older adults.

\section{Acknowledgement}

This project has received funding from the European Union's Horizon 2020 research and innovation programme under grant agreement No 693319

\section{References}

1. Bolton, M., Loneliness - the state we're in: A report of evidence compiled for the Caimpaign to End Loneliness. 2012, Age UK Oxfordshire.

2. Commission for Rural Communities, Social Isolation Experienced by Older Adults in Rural Communities, C.f.R. communities, Editor. 2012: Gloucester. p. 78.

3. Poscia, A., et al., Interventions targeting loneliness and social isolation among the older people: An update systematic review. Exp Gerontol, 2018. 102: p. 133-144.

4. Jones, R.B., et al., Older people going online: its value and before-after evaluation of volunteer support. J Med Internet Res, 2015. 17(5): p. e122.

5. Austin, J., et al., A Smart-Home System to Unobtrusively and Continuously Assess Loneliness in Older Adults. IEEE Journal of Translational Engineering in Health and Medicine, 2016. 4: p. 111.

6. Hill, R., L.R. Betts, and S.E. Gardner, Older adults' experiences and perceptions of digital technology: (Dis)empowerment, wellbeing, and inclusion. Computers in Human Behavior, 2015. 48: p. 415423.

7. Niehaves, B. and R. Plattfaut, Internet adoption by the elderly: employing IS technology acceptance 
theories for understanding the age-related digital divide. European Journal of Information Systems, 2014. 23(6): p. 708-726.

8. Rockmann, R. and H. Gewald. How Do IT-related Traits Drive the Internet Use of Mature Adults?

The Interplay of Curiosity and Control. in Proceedings of the 51st Hawaii International Conference on System Sciences. 2018.

9. Fox, G. and R. Connolly, Mobile health technology adoption across generations: Narrowing the digital divide. Information Systems Journal, 2018.

10.Barnard, Y., et al., Learning to use new technologies by older adults: Perceived difficulties, experimentation behaviour and usability. Comput. Hum. Behav., 2013. 29(4): p. 1715-1724.

11.Schreurs, K., A. Quan-Haase, and K. Martin, Problematizing the Digital Literacy Paradox in the Context of Older Adults' ICT Use: Aging, Media Discourse, and Self-Determination. Canadian Journal of Communication, 2017. 42(2): p. 359-377.

12. Sipior, J.C., B.T. Ward, and R. Connolly, The digital divide and t-government in the United States: using the technology acceptance model to understand usage. European Journal of Information Systems, 2017. 20(3): p. 308-328.

13. Philip, L., et al., The digital divide: Patterns, policy and scenarios for connecting the 'final few' in rural communities across Great Britain. Journal of Rural Studies, 2017. 54: p. 386-398.

14. Ashmore, F.H., J.H. Farrington, and S. Skerratt, Community-led broadband in rural digital infrastructure development: Implications for resilience. Journal of Rural Studies, 2017. 54: p. 408-425.

15. Boase, J., The Consequences of Personal Networks for Internet Use in Rural Areas. 2010. p. 12571267.

16. Saunders, E.J., Maximizing Computer Use Among the Elderly in Rural Senior Centers. Educational Gerontology, 2004. 30(7): p. 573-585.

17. Hakkarainen, P., 'No Good for Shovelling Snow and Carrying Firewood': Social Representations of Computers and the Internet by Elderly Finnish Non-users. New Media \& Society, 2012. 14(7): p. 1198-1215.

18. Hodge, H., et al., Using Internet technologies in rural communities to access services: The views of older people and service providers. Journal of Rural Studies, 2017. 54: p. 469-478.

19. Suopajarvi, T., Past experiences, current practices and future design. Technological Forecasting \& Social Change, 2015. 93: p. 112.

20. Quan-Haase, A., K. Martin, and K. Schreurs, Interviews with digital seniors: ICT use in the context of everyday life. Information, Communication \& Society, 2016: p. 1-17.

21. Bozan, K., B. Davey, and K. Parker, Social Influence on Health IT Adoption Patterns of the Elderly: An Institutional Theory Based Use Behavior Approach. Procedia Computer Science, 2015. 63(C): p. 517-523.

22. Orlikowski, W.J., Using Technology and Constituting Structures: A Practice Lens for Studying Technology in Organizations. Organization Science, 2000. 11(4): p. 404-428.

23. Orlikowski, W.J., Sociomaterial Practices: Exploring Technology at Work. 2007. p. 14351448.

24. Shove, E., The dynamics of social practice: everyday life and how it changes, ed. M. Pantzar and M. Watson. 2012: SAGE.

25. Shove, E., Converging Conventions of Comfort, Cleanliness and Convenience. Consumer Issues in Law, Economics and Behavioural Sciences, 2003. 26(4): p. 395-418.

26. Pantzar, M. and E. Shove, Temporal Rhythms as Outcomes of Social Practices. A Speculative Discussion. Ethnologia Europaea, 2010. 40(1): p. 19-29.

27. Shove, E., The Design of Everyday Life: Cultures of Consumption Series v. 5. 2007: Bloomsbury UK A \& C Black.

28. Pantzar, M. and E. Shove, Understanding innovation in practice: a discussion of the production and re-production of Nordic Walking. Technol. Anal. Strateg. Manage., 2010. 22(4): p. 447-461.

29. Frayling C., Research in art and design. Royal College of Art Research Papers, 1993. 1(1). London: Royal College of Art

30. Stappers, P.J. Doing design as a part of doing research. In R. Michel and K.T. Edelmann, eds., Design research now: essays and selected projects. Birkhäuser, Basel, 2007, 81-91.

31.Kemmis, S. and McTaggart, R. Participatory Action Research. In N.K. Denzin and Y.S. Lincoln, eds., Handbook of Qualitative Research. SAGE Publications, Inc, Thousand Oaks, 1994, 271-330.

32. Kolb, D.A. Management and the Learning Process. California Management Review 18, 3 (1976), 21-31.

33. Leitch, R. and Day, C. Action research and reflective practice: towards a holistic view. Educational Action Research 8, 1 (2000), 179-193.

34. McIntosh, P. Action Research and Reflective Practice: Creative and Visual Methods to Facilitate Reflection and Learning. Routledge, London, 2010.

35. Zuber-Skerritt, O., Kemmis, S., Altrichter, H., and McTaggart, R. The concept of action research. The Learning Organization 9, 3 (2002), 125-131. 